\title{
A CODED PROFILE METHOD FOR PREDICTING ACHIEVEMENT ${ }^{1}$
}

\author{
BENNO G. FRICKE \\ University of Michigan
}

Counselors and test users have often been advised to use the test profile in their attempt to understand students. Strictly speaking, this has never been done systematically when scores have been used for the prediction of academic achievement. The ability of the test interpreter to attach "armchair beta weights" to individual test variables, and to see significance in discrepancies in test scores varies considerably. When only test information is available, the grades predicted for a student with a particular profile should always be the same. Furthermore, there seems to be ample evidence $(9$, IO, I I) for believing that counselors, even with additional information (biographical data, interview impressions, etc.) are unable to improve the accuracy of actuarial predictions made from a multiple regression equation involving two test variables.

\section{Purpose}

The purpose of this paper is to present a simple objective method for doing what the test user attempts to do, and what multiple regression, discriminative function, and other statistical approaches cannot do; namely, consider simultaneously, rather than separately, the magnitude and the relation of two or more test scores. ${ }^{2}$ The assumption is that much information is lost when one score is used without simultaneously considering information obtainable from other scores; inter-score relationships of gestalt-like importance are largely ignored by

\footnotetext{
1 This paper is based on material prepared for a "measurement" course taught by the writer during the Winter Quarter, I955, at the University of Minnesota as part of his Ford Foundation Teaching-Intern Fellowship.

2 The writer $(2,3,4)$ recently developed an empirically validated personality inventory employing configural analysis of item responses for the prediction of academic achievement; in each item of the test three responses are analyzed and scored simultaneously. Research with these compound items established that it was frequently possible to predict the criterion even though the component statements were singly invalid.
} 
traditional statistical approaches. A recent review by Furst and Fricke (7) disclosed that pattern analysis of personality inventory scores has been fruitful in revealing personality adjustment.

It occurred to the writer while he was examining hundreds of student records that there was a marked tendency for students with similar profiles to receive the same academic grades. $\mathrm{He}$ felt that these profiles might be coded, and the results provide the basis for predicting academic achievement. It would seem that the mean honor point ratio (HPR) of all students having the same coded profile would be the best predicted HPR that could be made for each student.

\section{Method}

The method to be outlined might be called coded profile prediction. The steps involved are these:

I. Divide the score distribution for each predictor variable ( $a, b, c$, etc.) in to an arbitrary, but small number of categories. The categories need not be the same width but it may be wise to have approximately the same number of individuals in each. The categories for each variable are then coded (i.e., given a number I, 2, 3, 4, etc.).

2. Preserve the variables in the same order, (a, b, c, etc.) and tally the honor point ratios (HPR's) for each coded profile.

3. Compute the mean HPR for each coded profile (e.g., the mean HPR for coded profile $3-2-2-3$ is 1.05 ). When coded profiles contain very few cases some smoothing and extrapolating may be necessary.

4. Prepare a table of coded profiles showing the mean HPR for each profile.

5. Locate in the table for each student the HPR corresponding to his coded profile. This predicted HPR would appear to be the best that could be made.

One way of demonstrating the efficiency of this coded profile prediction method would be to compute on a new sample a product moment correlation coefficient between predicted and obtained HPR's and to compare it with the multiple correlation coefficient obtained when the same variables are used in a multiple regression equation.

With the profile approach almost all the available information 
is used; some is lost in setting up coded categories. However, if the sample is large, and many categories and few variables are used, a negligible loss results. It would seem that fulfilling the usual statistical assumptions (normal distribution uncorrelated variables, linearity of regression, etc.) is not necessary. In a sense this procedure is an extension of multiple cut-off methods.

\section{Results and Discussion}

Some results indicating the value of the method are available, although it has not been possible to complete each of the steps described above.

Entrance scores were obtained on $2,9^{8} 3$ freshman men who entered the College of Science, Literature, and the Arts (SLA) of the University of Minnesota the same year they were graduated from high school. These freshmen entered SLA during the years 1949 to 1954 . The criterion to be predicted is HPR earned at the end of three or less quarters of work. The centile distribution on each of the four predictors was arbitrarily divided into five or six categories as shown in Table $I$.

The mean centiles for these freshmen in SLA are as follows:

High School Rank......... 70 (high school graduates) American Council on Education

Psychological Examination. 72 (entering $\mathrm{U}$ of $\mathrm{M}$ freshmen) Cooperative English....... 65 (entering $U$ of $M$ freshmen) Ohio State Psychological Test. 39 (entering SLA freshmen)

In SLA all but the Ohio centile distribution are skewed negatively. The average Ohio centile for all SLA freshmen is 50, of course; however, men (mostly older), and women not

TABLE I

Centile Values in Each Category on Four Predictors

\begin{tabular}{ccccc}
\hline \multirow{2}{*}{ Category } & \multicolumn{4}{c}{ Predictor } \\
\cline { 2 - 5 } & $\begin{array}{c}\text { HSR } \\
\text { (a) }\end{array}$ & $\begin{array}{c}\text { ACE } \\
\text { (b) }\end{array}$ & $\begin{array}{c}\text { English } \\
\text { (c) }\end{array}$ & $\begin{array}{c}\text { Ohio } \\
\text { (d) }\end{array}$ \\
\hline I & $86-100$ & $91-100$ & $86-100$ & 7 I-I00 \\
2 & $76-85$ & $81-90$ & $76-85$ & $46-70$ \\
3 & $66-75$ & $7 \mathrm{I}-80$ & $66-75$ & $3 \mathrm{I}-45$ \\
4 & $5 \mathrm{I}-65$ & $5 \mathrm{I}-70$ & $51-65$ & $\mathrm{I}-30$ \\
5 & $\mathrm{I}-50$ & $26-50$ & $\mathrm{I}-50$ & $\mathrm{I}-15$ \\
6 & & $\mathrm{I}-25$ & & \\
\hline
\end{tabular}


meeting the requirements for inclusion in the sample score significantly higher. Women also rank considerably higher on HSR and English. The sample was restricted to a homogeneous sample of men to eliminate known contaminating factors.

Since the distribution on each variable is not rectangular and since the variables are intercorrelated $(.3$ to .7$)$ the number of cases falling into each possible coded profile is not the same. While there are almost one hundred students having all their scores in the top category (coded profile I-I-I-I) there are no students in coded profiles $4^{-2-I-I}, 3^{-2-I-I}$, nor in many others.

Three kinds of evidence of the predictive power of the coded profile prediction method are presented. The median HPR of the 24 students having all their centiles above 96 (not coded profile I-I-I-I) is $2.78 .^{3}$ Generally only the $H S R$ and the Ohio have significant beta weights when combined with the $A C E$ and English in a multiple regression equation. A measure of "academic personality" obtained from the Opinion, Attitude and Interest Survey (OAIS), however, did have a significant beta weight when used with the other four predictors (2). Nevertheless the writer has not made, or seen, regression equation predictions above 2.3 on SLA students. That is, the regression equation predictions do not exceed 2.3 when the raw scores equivalent to centiles of 100 on all predictors are used. It is clear that the profile approach has held information which was lost by the multiple regression approach. Possibly the variables which do not have a significant beta weight contribute valid variance or serve as suppressor variables (5).

The mean HPR of the 49 students having average scores on each of the variables (profiles where no centile is more than four from the mean centile) is .96 ; this is less than the mean HPR of I.IO for all freshman men. An examination of the mean entrance centiles may provide a partial explanation for this. The average male SLA freshman may be an "underachiever." Although he exceeds approximately 72 per cent of the University freshmen in scholastic aptitude $(A C E)$ he was able to surpass only 70 per cent of his high school classmates

${ }^{3}$ At the University of Minnesota the honor points assigned for each credit are: $A, 3 ; B, 2 ; C, I ; D$ and $F, O$. 
in achievement. The average male high school graduate has an HSR of approximately 44, and an $A C E$ score higher than 25 per cent of the University freshmen (I). The discrepancy between the ACE and the English centiles might also be interpreted as reflecting underachievement.

Perhaps much more startling was the discovery that when two coded profile groups were selected on the basis of having similar HSR, English, and Ohio centiles, but widely divergent ACE's, their HPR's differed-but not in the statistically expected direction. These two subgroups were selected because the writer has been especially interested in studying students who under- and over-achieve academically in relation to their tested $A C E$.

The mean HPR of the 35 students with the 2-5-3-3 profile (see Table I) is I.27; the mean HPR of the 5 I students with the 2-I-3-3 profile is $\mathrm{x} .08$. The unbiased standard deviations are .32 and .47 , respectively. The Behrens-Fisher test (8) for the significance of differences between means when variances are unequal showed that the difference in means of .I9 was significant at the five per cent level. Normally multiple regression equations would predict higher HPR's for the 2-I-3-3 group, but actually their mean HPR is lower than that of the 2-5-3-3 group. A negative relation exists between ACE scores and HPR when three other scores are equal and the four scores are treated simultaneously. In the subgroups a high $\mathrm{ACE}$ score is an unfavorable prognostic sign!

One possible explanation for the latter finding is that the $A C E$ is a rather good test of intellectual potential or capacity; that the personality factors making for under- and overachievement are largely responsible for the difference in HPR's, and partly responsible for the magnitude of the other three scores. The profile pictures not only the student's ability and achievement, but also, by the relation of the test scores, his habits of work, and other psychological dimensions. The four scores provide a personality-ability-achievement profile.

\section{Conclusions and Implications}

From these findings it can be seen that it is possible for a variable that correlates positively with a criterion when treated 
separately, to correlate negatively with the criterion when treated simultaneously with several other variables that are approximately equal. It is also possible that certain variables may not contribute significantly to the multiple correlation coefficient, but may aid considerably in predicting the criterion when the coded profile prediction method is followed. In the multiple regression equation each variable has a constant beta weight. For an individual, the contribution of a particular score is not conditioned by the magnitude and relation of the other scores. Actually what might be called a "dynamic suppressor variable relationship" may be operating in the coded profile prediction method. Perhaps in order for a particular predictor to be able to exert its influence on the predictand, specific amounts of other predictors must be present. The writer has presented elsewhere (6) a method for predicting the behavior of psychologists from two singly invalid predictors.

It would seem that the method described in this paper might profitably be used to predict academic achievement with the Differential Aptitude Tests, the Wechsler-Bellevue Scales, the Primary Mental Abilities Test, and other tests having reliable part scores. Those interested in furthering the development of psychometrics may find it worth while to consider simultaneously two or more bits of information about an individual. This applies not only to the study of test scores but also to the study of item responses.

\section{Summary}

This paper presented an objective method for using the information in a test profile for the prediction of academic achievement. ${ }^{4}$ The rationale underlying the coded profile prediction method is similar to the "birds of a feather flock to-

\footnotetext{
${ }^{4}$ While the emphasis in this paper has been on increasing the accuracy with which the critericn may be predicted, it is relevant to point out that after the researcher has demonstrated the value of a particular method, test, etc., he should assist the consumers of research (e.g., selection officers and counselors) to use the information in such a way as to reduce or eliminate the predictive value of the method, test, etc. Questions concerning the desirability of high predictability are developed in a recent article on "Prediction, Selection, Mortality, and Quality Control." College and University: Fournal of the American Association of Collegiate Registrars and Admissions Officers, XXXII (1956), 34-52.
} 
gether" point of view, which is that students with the same test scores get the same grades. The marked differences in the approach and results between the suggested method and traditional statistical methods were pointed out. Perhaps the major objection to the coded profile prediction method is that large samples are necessary, and much clerical work is involved.

\section{REFERENCES}

I. Berdie, R. F., Layton, W. L., and Hagenah, Theda. A Manual for the State-Wide Testing Programs of Minnesota: Using Tests in Counseling. Minneapolis: Student Counseling Bureau, University of Minnesota, 1953.

2. Fricke, B. G. The Development of an Empirically Validated Personality Test Employing Configural Analysis for the Prediction of Academic Achievement. Unpublished doctor's dissertation, University of Minnesota, I954.

3. Fricke, B. G. The Opinion, Attitude and Interest Suroey. Minneapolis: Investors Diversified Services, I955.

4. Fricke, B. G. "A Configural-Content-Intensity Item for Personality Measurement." Educational and Psychological Measurement, XVI (1956), 54-62.

5. Fricke, B. G. "Response Set as a Suppressor Variable in the OAIS and MMPI." Fournal of Consulting Psychology, XX (1956), I6I-I6g.

6. Fricke, B. G. "Configurations of Contributing Psychologists." American Psychologist, XII (1957), in press.

7. Furst, E. J. and Fricke, B. G. "Development and Applications of Structured Tests of Personality." Review of Educational Research, XXVI (1956), 26-55.

8. Johnson, P. O. Statistical Methods in Research. New York: Prentice-Hall, I 949.

9. Kelly, E. L. and Fiske, D. W. The Prediction of Performance in Clinical Psychology. Ann Arbor: University of Michigan Press, I95I.

Io. Melton, R. M. A Comparison of Clinical and Actuarial Methods of Prediction with an Assessment of the Relative Accuracy of Different Clinicians. Unpublished doctor's dissertation, University of Minnesota, 1952.

I I. Sarbin, T. R. The Relative Accuracy of Clinical and Statistical Predictions of Academic Success. Unpublished doctor's dissertation, Ohio State University, I94I. 